\title{
Transformative Wissenschaft: zurück ins Labor
}

\begin{abstract}
Transformative Wissenschaft versteht sich als Katalysator für gesellschaftliche Veränderungsprozesse. Sie musste sich in den vergangenen Jahren innerwissenschaftlicher Kritik und Dekonstruktion stellen. Daneben muss sie sich aber auch an ihrer gesellschaftlichen Wirkung messen lassen. Als Zwischenbilanz aus fünfJahren Debatte um die transformative Wissenschaft halten wir fest: Die nächste Phase in der Weiterentwicklung transformativer Wissenschaft braucht Orte, an denen ihre gesellschaftlich produktive Wirkkraft erfahrbar wird und erprobt werden kann.
\end{abstract}

Mandy Singer-Brodowski, Uwe Schneidewind

Transformative science: back to the lab | GAIA 28/1 (2019):26-28

Keywords: real-world laboratories, science policy, transdisciplinarity, transformative science

$\mathrm{D}$ as Konzept der transformativen Wissenschaft knüpft an den seit Ende der 1990er Jahre etablierten Diskurs um eine transdisziplinäre Forschung an. Ihr Anspruch ist es, diesen Diskurs durch eine grundlegende Debatte über veränderte Förderbedingungen, die Rolle zivilgesellschaftlicher Akteure und das Verhältnis von Wissenschaft und Gesellschaft zu beleben und mit Vorschlägen für politische Innovationen zur Förderung der Nachhaltigkeitsforschung zu untersetzen. Die transformative Wissenschaft zielt darauf, politische Aufmerksamkeit dafür zu schaffen, dass eine gesellschaftsorientierte Forschung in einem strukturkonservativen, primär disziplinär geprägten Wissenschaftssystem institutionelle Unterstützung braucht. Auf institutioneller Ebene geht es also um etwas, was für die individuelle Ebene als eine „Destabilisierung der Beharrungskräfte“ (Koller 2011, S.71) beschrieben wird und damit um die Voraussetzung transformativer Lernprozesse (Singer-Brodowski 2016).

Wir haben das Konzept der transformativen Wissenschaft im Jahr 2013 in unserem gleichnamigen Buch vorgestellt (Schneidewind und Singer-Brodowski 2013). Vor zehn Jahren war die Vorläuferpublikation Nachhaltige Wissenschaft erschienen (Schneidewind 2009). Wir nutzen daher nun die Gelegenheit, um eine Zwischenbilanz zu ziehen: In welcher Form wurde das Konzept einer transformativen Wissenschaft rezipiert? Welche Wirkungen hatte die Publikation? Und wie geht es weiter mit der transformativen Wissenschaft?

Dr. Mandy Singer-Brodowski | Freie Universität Berlin | Institut Futur | Fabeckstr. 37 | 14195 Berlin | Deutschland | +493083861337 |

s-brodowski@institutfutur.de

Prof. Dr. Uwe Schneidewind I Wuppertal Institut für Klima, Umwelt, Energie GmbH | Döppersberg 19 | 42103 Wuppertal | Deutschland |

+492022492100 | uwe.schneidewind@wupperinst.org

(c) 2019 M. Singer-Brodowski, U. Schneidewind; licensee oekom verlag.

This is an article distributed under the terms of the Creative Commons Attribution License

(http://creativecommons.org/licenses/by/3.0).

https://doi.org/10.14512/gaia.28.1.8

Submitted February 3, 2019; revised version accepted February 12, 2019

\section{Die innerwissenschaftliche Rezeption der transformativen Wissenschaft}

Mit dem Konzept der transformativen Wissenschaft ging der Versuch einher, die Verhältnisse in einem sich zunehmend standardisierenden und verengenden Wissenschaftssystem zum Schwingen zu bringen und die grundsätzlichen Bedingungen für transdisziplinäre Nachhaltigkeitsforschung zu verbessern. Es ging nicht darum, das bestehende Wissenschaftssystem zu ersetzen, wie es etwa Strunz und Gawel (2017) deuteten.

Gleichzeitig zielte unser Buch (Schneidewind und Singer-Brodowski 2013) darauf, den zahlreichen Akteuren, die sich an ihren Hochschulen für eine konkrete Nachhaltigkeitstransformation engagieren, einen Kompass an die Hand zu geben und sie in der „Kunst gesellschaftlichen Wandels“ (Schneidewind 2018) anzuleiten: konkret in der Praxis der Veränderung der eigenen Hochschule und des wissenschaftlichen Handlungsraums.

Die von der transformativen Wissenschaft pointiert formulierten Forderungen - vielleicht auch der provokative Titel des Buchs selbst - berührten das Selbstverständnis von Wissenschaftler(inne)n sowie Fragen institutioneller Veränderungspotenziale und Ressourcenverteilungen im Wissenschaftssystem.

Dir Wirkung dieser Intervention ist durchaus beachtlich: Das Buch und die resultierende Debatte haben im deutschen Wissenschaftssystem die Diskussion über ein neues Verhältnis von Wissenschaft und Gesellschaft angeregt. Die von einer Veröffentlichung des DFG-Präsidenten Peter Strohschneider (Strohschneider 2014) ausgehende Kontroverse in GAIA (Grunwald 2015, Rohe 2015, Schneidewind 2015, von Wissel 2015) ist ein besonders lebendiger Ausdruck dafür.

Die Rezeption des Buchs reicht aber darüber hinaus. Dies zeigt eine Analyse seiner bisherigen Zitationen. ${ }^{1}$ Zentrale Referenzen beziehen sich sowohl auf die grundlegenden Überlegungen zum

\footnotetext{
1 Eine Abfrage bei Google Scholar am 6.12.2018 ergab 273 Zitationen; abzüglich aller Selbstzitationen blieben 237 Zitationen.
} 
Verhältnis von Wissenschaft und Gesellschaft in der transformativen Wissenschaft (zum Beispiel Wolf et al. 2015) als auch auf institutionelle Transformationsanforderungen einzelner Hochschulen (zum Beispiel Nölting et al. 2016). Weiterhin zeigt die Zitationsanalyse, dass das Buch in unterschiedlichen Disziplinen (Soziologie, Stadtplanung, Erziehungswissenschaft, Wirtschaftswissenschaft etc.) und Forschungsfeldern (Verbraucherforschung, Entwicklungszusammenarbeit, Agrarforschung, Klimaanpassung) produktiv aufgegriffen wird.

Der scientific impact dieses deutschsprachigen Buchs kann sich daher durchaus sehen lassen. Strohschneiders Auseinandersetzung mit dem Konzept einer transformativen Wissenschaft (Strohschneider 2014) und die darauf aufbauende Debatte in GAIA haben in vielerlei Hinsicht Anstöße zur Klärung des Verhältnisses von Wissenschaft und Gesellschaft gegeben: zur Rolle von Wissenschaft in Demokratien (Strunz und Gawel 2017, Grunwald 2018) oder zur Kohärenz in den Förderbedingungen (Kläy und Schneider 2015). Über die Strohschneider-Debatte hinaus zeigt sich in den Zitationen, dass das Konzept zumeist positiv aufgenommen wurde. Auch in Artikeln, die den Strohschneider-Beitrag und seine Repliken thematisieren, jedoch nicht Teil der GAIA-Debatte sind, offenbart sich eine differenzierte und kritische Auseinandersetzung mit den wesentlichen Argumenten (Mielke et al. 2016, Scholz 2017, Opielka 2016).

\section{Die gesellschaftliche Wirkung der transformativen Wissenschaft}

Eine Bilanz der transformativen Wissenschaft darf sich aber nicht auf die innerwissenschaftliche Rezeption beschränken, sondern muss sich auch an erweiterten Impact-Kriterien messen lassen. Im Bewusstsein, dass die Suche nach geeigneten Kriterien für die Erfassung des societal impact von Forschung nicht abgeschlossen ist, soll der Impact des Konzepts transformative Wissenschaft doch an einigen Beispielen verdeutlicht werden.

Große Resonanz fand das Buch in zivilgesellschaftlichen Organisationen und in der Wissenschaftspolitik ausgewählter deutscher Bundesländer, besonders in Nordrhein-Westfalen und in Baden-Württemberg:

- In Baden-Württemberg wurden - unter anderem auf Basis des in der transformativen Wissenschaft angelegten Konzepts „Reallabor“ und den darauf aufbauenden Empfehlungen einer Expertenkommission - in zwei Förderrunden 18 Millionen Euro für Nachhaltigkeitsforschung in Reallaboren vergeben. Der politische Effekt solcher strukturbildenden Maßnahmen an den jeweiligen Hochschulen ist von hoher Bedeutung. Dies gilt, auch wenn wir den Argumenten für eine Entschleunigung und stärkere Systematisierung der Praxis transformationsorientierter Forschung (Jaeger-Erben et al. 2018) durchaus folgen können. Die positive politische Bewertung einer neuen Forschungsperspektive (Landtag BW 2018) schafft nämlich erst die Ressourcen für Forschungsprojekte, in denen genau jene Diskurse über die verschiedenen Varianten gesellschaftsorien- tierter Nachhaltigkeitsforschung vorangetrieben werden können.

- Die Positionierungsentscheidungen einzelner Hochschulen wurden durch das Konzept einer transformativen Wissenschaft inspiriert oder begründet. So hat die Universität Potsdam im aktuellen Hochschulentwicklungsplan ihre Third-MissionOrientierung verankert und darin die transformative Wissenschaft hervorgehoben (Universität Potsdam 2014, S. 58). Die Hochschule für nachhaltige Entwicklung Eberswalde hat sich in ihrer Transferstrategie (Hochschule Eberswalde 2016) an der transformativen Wissenschaft ausgerichtet und im Rahmen des Verbundprojekts HOCHN für die Debatte um ein gesellschaftsorientiertes Transfer-Verständnis ein eigenes Konzept für Nachhaltigkeitstransfer vorgelegt (Nölting et al. 2018).

- Die in der transformativen Wissenschaft angelegten Forderungen nach einer Demokratisierung der Wissenschaft haben auch in der Diskussion über erweiterte Partizipationsmöglichkeiten, zum Beispiel in der Krebsforschung (Müller-Jung 2018), ihren Ausdruck gefunden.

Diese wissenschaftspolitische Wirkmächtigkeit des Konzepts erklärt vermutlich auch die Vehemenz der Reaktionen einzelner prominenter Vertreter(innen) des Wissenschaftssystems.

Die institutionellen Impulse ins deutsche Wissenschafts- und Hochschulsystem machen zugleich deutlich, dass eine rein theoretisch-konzeptionelle Beurteilung der (Un-)Schärfen der transformativen Wissenschaft zu kurz greift. Es ist ja gerade diese rein innerwissenschaftliche Selbstbezüglichkeit, die das Konzept aufbrechen möchte. Die in den GAIA-Beiträgen formulierte Kritik an der transformativen Wissenschaft hat wichtige Hinweise auf Möglichkeiten zur konzeptionellen und theoretischen Schärfung gegeben. Dieser Modus innerwissenschaftlicher Kritik und Dekonstruktion muss die transformative Wissenschaft weiter begleiten, darf aber nicht die einzige Ebene der Bewertung bleiben. Diese muss vielmehr ergänzt werden um die Bewertung ihres konkreten gesellschaftlichen Impacts, das heißt: der Umsetzbarkeit und erfolgreichen Weiterentwicklung der im Buch vorgestellten institutionellen Gestaltungsideen.

\section{„Zurück ins Labor": Wie weiter mit der transformativen Wissenschaft?}

Die nächste Phase transformativer Wissenschaft braucht daher Orte und Arenen, in denen ihre gesellschaftlich produktive Wirkkraft erfahrbar wird und erprobt werden kann. Reallabore, insbesondere langfristig und über mehrere Ebenen - von Haushalten über Quartiere bis zu ganzen Städten - angelegte urbane Reallabore (siehe Schneidewind et al. 2018), sind dafür die vermutlich beste Form. Sie können zudem auch einer drohenden Verflachung des Reallabor-Konzepts entgegenwirken. Eine solche Verflachung droht, wenn etwa einzelne unverbundene und temporäre Experimente in einer Stadt schon als Reallabor verstanden werden. Denn Reallabore waren immer als Orte gedacht, in denen sich durch co- 
design und gemeinsame Wissensproduktion konkrete und langfristige gesellschaftliche Veränderungsprozesse vollziehen.

Auch das für eine transformative Wissenschaft zentrale Konzept „transformativen Lernens“ über die gesamte menschliche Lebensspanne und alle Bildungsinstitutionen hinweg muss künftig sichtbarer und greifbarer werden. Lebendige Formen transformativen Lernens, die sich insbesondere im Kontext non-formaler Lernorte eröffnen (Singer-Brodowski im Erscheinen) können das Lernen auf Hochschulebene inspirieren.

Auf den Punkt gebracht: Nach fünf (oder zehn) Jahren öffentlicher wissenschaftspolitischer Debatten gilt es, die transformative Wissenschaft ins „Labor“ zurückzuschicken und dort ihre Wirkung konkret sichtbar zu machen. Orte, an denen die produktiv verändernde Kraft einer transformativen Wissenschaft erfahrbar wird, werden dann auch die konzeptionellen Debatten über die Art und den Sinn eines veränderten Wissenschafts- und Gesellschaftsverhältnisses beflügeln.

\section{Literatur}

Grunwald, A. 2015. Transformative Wissenschaft - eine neue Ordnung im Wissenschaftsbetrieb? GAIA 24/1: 17-20. DOI: 10.14512/gaia.24.1.5.

Grunwald, A. 2018. Transformative Wissenschaft als honest broker? Das passt! GAIA 27/1: 113-116. DOI: 10.14512/gaia.27.1.4.

Hochschule Eberswalde (Hochschule für nachhaltige Entwicklung Eberswalde). 2016. Transferstrategie. Eberswalde. www.hnee.de/_obj/ F2E4A8B2-66E3-4D13-AA12-1270ABB17F24/outline/16101290_ Transferbroschuere_WEB.pdf (abgerufen 08.02.2019).

Jaeger-Erben, M, E. Nagy, M. Schäfer, E. Süßbauer, J. Zscheischler. 2018. Von der Programmatik zur Praxis: Plädoyer für eine Grounded Theory transformationsorientierter Forschung. GAIA 27/1:117-121. DOI: 10.14512/gaia.27.1.5.

Kläy, A, F. Schneider. 2015. Zwischen Wettbewerbsfähigkeit und nachhaltiger Entwicklung: Forschungsförderung braucht Politikkohärenz. GAIA 24/4: 224-227. DOI: 10.14512/gaia.24.4.4.

Koller, H.-C. 2011. Bildung anders denken. Einführung in die Theorie transformatorischer Bildungsprozesse. Stuttgart: W. Kohlhammer.

Landtag BW (Landtag von Baden-Württemberg). 2018. Reallabore als neues Forschungsformat: Ein baden-württembergischer Erfolg. Drucksache 16/4881. 28.09.2018. www.landtag-bw.de/files/live/sites/LTBW/files/dokumente/ WP16/Drucksachen/4000/16_4881_D.pdf (abgerufen 08.02.2019).

Mielke, J., H. Vermaßen, S. Ellenbeck, B. F. Milan, C. Jaeger. 2016. Stakeholder involvement in sustainability science. A critical view. Energy Research Q Social Science 17: 71-81. DOI: 10.1016/j.erss.2016.04.001.

Müller-Jung, J. 2018. Krebsforschung lässt Bürger mitreden. Frankfurter Allgemeine Zeitung, 24.11.2018. www.faz.net/aktuell/wissen/krebsforschungschafft-patientenbeirat-und-laesst-buerger-mitreden-15899242.html (abgerufen 08.02.2019).

Nölting, B., J. Pape, B. Kunze. 2016. Nachhaltigkeitstransformation als Herausforderung für Hochschulen - Die Hochschule für nachhaltige Entwicklung Eberswalde auf dem Weg zu transdisziplinärer Lehre und Forschung. In: Forschung für Nachhaltigkeit an deutschen Hochschulen. Herausgegeben von W. Leal Filho. Wiesbaden: Springer Spektrum. 131-147.

Nölting, B. et al. 2018. Transfer für nachhaltige Entwicklung an Hochschulen. Nachhaltigkeit an Hochschulen: entwickeln - vernetzen - berichten (HOCHN). Eberswalde. www.hochn.uni-hamburg.de/-downloads/handlungsfelder/ transfer/hoch-n-leitfaden-transfer-fuer-nachhaltige-entwicklung-anhochschulen.pdf (abgerufen 08.02.2019).

Opielka, M. 2016. Soziale Nachhaltigkeit aus soziologischer Sicht. Soziologie 45/1: 33-46. http://publikationen.soziologie.de/index.php/soziologie/ article/download/852/1107 (abgerufen 08.02.2019).
Rohe, W. 2015. Vom Nutzen der Wissenschaft für die Gesellschaft: Eine Kritik zum Anspruch der transformativen Wissenschaft. GAIA 24/3: 156-159. DOI: 10.14512 /gaia.24.3.5.

Schneidewind, U. 2009. Nachhaltige Wissenschaft. Plädoyer für einen Klimawandel im deutschen Wissenschafts- und Hochschulsystem. Marburg: Metropolis.

Schneidewind, U. 2015. Transformative Wissenschaft - Motor für gute Wissenschaft und lebendige Demokratie. GAIA 24/2: 88-91. DOI: 10.14512/gaia.24.2.5.

Schneidewind, U. 2018. Die große Transformation. Eine Einführung in die Kunst gesellschaftlichen Wandels. Unter Mitarbeit von M. Fischedick, S. Lechtenböhmer, S. Thomas. Frankfurt am Main: Fischer (Forum für Verantwortung).

Schneidewind, U., K. Augenstein, F. Stelzer, M. Wanner. 2018. Structure Matters: Real-world laboratories as a new type of large-scale research infrastructure. A framework inspired by Giddens' structuration theory. GAIA 27/S1: 12-17.

Schneidewind, U., M. Singer-Brodowski. 2013. Transformative Wissenschaft. Klimawandel im deutschen Wissenschafts- und Hochschulsystem. Marburg: Metropolis.

Scholz, R. 2017. The normative dimension in transdisciplinarity, transition management, and transformation sciences. New roles of science and universities in sustainable transitioning. Sustainability 9/6: 991. DOI: 10.3390/su9060991.

Singer-Brodowski, M. 2016. Transformative Bildung durch transformatives Lernen. Zur Notwendigkeit der erziehungswissenschaftlichen Fundierung einer neuen Idee. Zeitschrift für internationale Bildungsforschung und Entwicklungspädagogik 39/1:13-17.

Singer-Brodowski, M. Im Erscheinen. Bildung für nachhaltige Entwicklung im Bereich des non-formalen und informellen Lernens. In: Pfade der Transformation. Die Diffusion von Bildung für nachhaltige Entwicklung in Deutschland. Herausgegeben von M. Singer-Brodowski, N. Etzkorn, T. Grapentin-Rimek. Opladen: Barbara Budrich.

Strohschneider, P. 2014. Zur Politik der Transformativen Wissenschaft. In: Die Verfassung des Politischen. Festschrift für Hans Vorländer. Herausgegeben von A. Brodocz et al. unter Mitarbeit von Hans Vorländer. Wiesbaden: Springer VS. 175-192.

Strunz, S., E. Gawel. 2017. Transformative Wissenschaft: eine kritische Bestandsaufnahme der Debatte. GAIA 26/4: 321-325. DOI: 10.14512/gaia.26.4.8.

Universität Potsdam. 2014. Hochschulentwicklungsplan 2014-2018. Potsdam. www.uni-potsdam.de/fileadmin01/projects/up-entdecken/docs/ Universitaet_Potsdam_Hochschulentwicklungsplan_2014-2018.pdf (abgerufen 08.02.2019)

von Wissel, C. 2015. Die Eigenlogik der Wissenschaft neu verhandeln: Implikationen einer transformativen Wissenschaft. GAIA 24/3: 152-155. DOI: 10.14512/gaia.24.3.4.

Wolf, B. M., A.-M. Häring, J. Heß. 2015. Strategies towards evaluation beyond scientific impact. Pathways not only for agricultural research. Organic Farming 1/1. DOI: 10.12924/of2015.01010003.

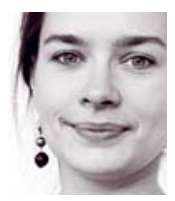

\section{Mandy Singer-Brodowski}

Geboren 1985 in Gera. Studium der Erziehungswissenschaft in Erfurt. Promotion an der Fakultät Nachhaltigkeit der Leuphana Universität Lüneburg. 2012 bis 2015 wissenschaftliche Referentin des Präsidenten im Wuppertal Institut für Klima, Energie, Umwelt GmbH. Seit 2016 Koordination des Monitorings des UNESCO-Weltaktionsprogramms Bildung für nachhaltige Entwicklung am Institut Futur, Fachbereich Erziehungswissenschaft und Psychologie der Freien Universität Berlin.

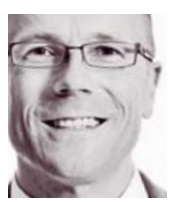

\section{Uwe Schneidewind}

Geboren 1966 in Köln. Studium der Betriebswirtschaftslehre in Köln. Promotion und Habilitation an der Universität St. Gallen. 1998 bis 2010 Professor für Betriebswirtschaftslehre an der Universität Oldenburg (2004 bis 2008 deren Präsident). Seit 2010 Präsident des Wuppertal Instituts für Klima, Umwelt, Energie $\mathrm{GmbH}$. Professor für Sustainable Transition Management an der Universität Wuppertal. Mitglied im Wissenschaftlichen Beirat der Bundesregierung Globale Umweltveränderungen (WBGU) und im GAIA-Aufsichtsrat. 